PROCEEDINGS OF THE

AMERICAN MATHEMATICAL SOCIETY

Volume 127, Number 6, Pages 1633-1639

S 0002-9939(99)04717-6

Article electronically published on February 18, 1999

\title{
PRESENTATION OF CRITICAL MODULES OF GK-DIMENSION 2 OVER ELLIPTIC ALGEBRAS
}

\author{
K. AJITABH AND M. VAN DEN BERGH \\ (Communicated by Lance W. Small)
}

\begin{abstract}
We show that critical modules of Gelfand-Kirillov dimension 2 and multiplicity $d$ over an elliptic algebra have (up to modules of lower GKdimension and shifting) a presentation by $d \times d$-matrices of linear forms. In the language of non-commutative algebraic geometry this amounts to a generic description of "curves" of degree $d$ in a projective quantum plane.
\end{abstract}

\section{Introduction}

Throughout the paper, $k$ will be an algebraically closed field and $A$ will be a three-dimensional Artin-Schelter regular $k$-algebra with three generators [5]. Thus $A$ is a graded $k$-algebra having the Hilbert series of a commutative polynomial algebra with three generators, and has some other desirable homological properties as in the commutative case (for this reason one sometimes refers to $A$ as a quantum polynomial ring in three variables, which defines a quantum plane).

In [3] the first author initiated the study of graded modules of Gelfand-Kirillov (GK) dimension 2 over $A$, the motivation being that these somehow correspond to "curves" in the corresponding quantum plane. Some important questions remained open about these modules. In the following theorem we answer one of these open questions.

Theorem 1.1. Assume that in the triple $(E, \sigma, \mathcal{L})$ associated to $A[6], E$ is a smooth elliptic curve and $\sigma$ is a translation of infinite order. Then every graded critical A-module of GK-dimension 2 and multiplicity d has, up to GK-equivalence and shifting, a resolution of the form

$$
0 \rightarrow A^{d}(-1) \rightarrow A^{d} \rightarrow M \rightarrow 0 .
$$

Two modules are called GK-equivalent if they have same GK-dimension and are isomorphic modulo modules of lower GK-dimension (Definition 2.1).

We conjecture that the hypotheses on the triple $(E, \sigma, \mathcal{L})$ are in fact unnecessary for the previous theorem to hold.

It was shown by the first author in [1] that Theorem 1.1 is true provided every critical normal $A$-module of GK-dimension 2 maps surjectively onto a point

Received by the editors September 23, 1997.

1991 Mathematics Subject Classification. Primary 16D50, 16E10, 18G10.

Key words and phrases. Elliptic algebras, quantum planes, regular algebra, critical modules, Cohen-Macaulay modules.

The second author is a senior researcher at the FWO. 
module (see below for definitions). Unfortunately it is not known if this is true. However in this note we succeed in proving Theorem 1.1, using an idea from [1] while sidestepping this open problem.

Thus, a generic way to describe "curves in a quantum plane" is by $d \times d$ matrices with linear entries. Comparing with the commutative case, one could also hope to be able to to show that, up to modules of lower GK-dimension and shifting, every graded critical $A$-module of GK-dimension 2 and multiplicity $d$ has a resolution of the form

$$
0 \rightarrow A(-d) \rightarrow A \rightarrow M \rightarrow 0
$$

This would mean that we could describe the curve corresponding to $M$ by one element of degree $d$. However it is not known if this is true.

In this note all modules are tacitly assumed to be graded and homomorphisms between them degree-preserving. Unless stated otherwise, the term A-module will mean a finitely generated graded right A-module.

\section{Preliminaries on $A$-modules of GK-dimension 2}

In this section we make no hypotheses on the triple $(E, \sigma, \mathcal{L})$, associated to $A$.

Let gr $-A$ be the category of all finitely generated graded right $A$-modules, and let $(\mathrm{gr}-A)_{i}$ be the full subcategory of $\mathrm{gr}-A$ consisting of modules of GK-dimension at most $i$. It is clear that $(\mathrm{gr}-A)_{i}$ is a Serre subcategory of $\mathrm{gr}-A$.

Definition 2.1. Two $A$-modules are called GK-equivalent if they have the same GK-dimension, say $i$, and their images in the quotient category $\mathrm{gr}-A /(\mathrm{gr}-A)_{i-1}$ are isomorphic.

For nice rings, the study of $A$-modules up to GK-equivalence amounts to studying the indecomposable injective modules over $A$. See [2], [10] for applications to the residue-complex. In this paper our main interest will be the study of critical $A$ modules of GK-dimension 2, up to GK-equivalence.

It is well known that $A$ is Cohen-Macaulay and Auslander-regular (see [9] for definitions of these notions, and [9], [7] for proofs). This implies that the homological behaviour of $A$-modules is very close to that in the commutative case. Thus we will leave the routine proofs of such homological properties to the reader. It is in particular elementary to prove that any object in $(\mathrm{gr}-A)_{2} /(\mathrm{gr}-A)_{1}$ is represented by a Cohen-Macaulay module $M$ of GK-dimension 2. Here, Cohen-Macaulay means by definition that $\operatorname{Ext}_{A}^{i}(M, A)=0$ unless $i=3-\operatorname{GKdim}(M)$. It is convenient to say that a module is normal if it is Cohen-Macaulay and if $M_{n}=0$ if and only if $n<0$.

A Cohen-Macaulay module $M$ of GK-dimension 2 has a minimal graded projective resolution of the form

$$
0 \longrightarrow A\left(-j_{1}\right) \oplus \ldots \oplus A\left(-j_{r}\right) \longrightarrow A\left(-i_{1}\right) \oplus \ldots \oplus A\left(-i_{r}\right) \longrightarrow M \longrightarrow 0,
$$

where the indices $i_{\nu}, j_{\nu}$ indicate the shifts of the module. As a minimal projective resolution is unique up to (non-unique) isomorphism, the sequence of indices $\tau=\left(i_{1}, \ldots, i_{r} ; j_{1}, \ldots, j_{r}\right)$ appearing in $(2.1)$ is unique if we arrange the sequences $i_{1}, \ldots, i_{r}$ and $j_{1}, \ldots, j_{r}$ in non-decreasing order. In [3] the sequence $\tau$ was called the type of $M$. From (2.1) it easily follows that if $M$ is an arbitrary $A$-module of GK-dimension 2, then $\operatorname{dim} M_{n}=d n+c$, for large $n$. We call $d$ the multiplicity of $M$. Recall that a module $M$ is called critical if it has no proper quotient of the same 
GK-dimension as $M$. Thus, a simple object in $(\mathrm{gr}-A)_{2} /(\mathrm{gr}-A)_{1}$ is represented by a critical Cohen-Macaulay module $M$ of GK-dimension 2. Recall the following finiteness theorem [3, Corollary 3.8].

Theorem 2.2. There are only finitely many types of critical normal A-modules of GK-dimension 2 with a given multiplicity.

It is a combinatorial problem to write down all possible types for a given multiplicity: in multiplicity 1 there is only possible type, namely $(0 ; 1)$ corresponding to line modules; in multiplicity 2 , there are two possible types: $(0 ; 2)$ and $(0,0 ; 1,1)$; in multiplicity 3 , there are four: $(0 ; 3),(0,0: 1,2),(0,1 ; 2,2)$ and $(0,0,0 ; 1,1,1)$.

Below we improve certain results from [7], [3] on Hilbert functions of normal modules of GK-dimension 2.

Lemma 2.3. Let $M$ be a critical normal A-module of GK-dimension 2 and multiplicity $d$. Then $M$ has a minimal projective resolution of the form

$$
0 \rightarrow A^{d}(-1) \rightarrow A^{d} \rightarrow M \rightarrow 0
$$

if and only if the Hilbert function of $M$ is $h_{M}(n)=d n+d$ for all $n \geq 0$.

Proof. It is simple to compute the Hilbert function of a module $M$ with presentation $(2.2): h_{M}(n)=d\left[h_{A}(n)-h_{A}(n-1)\right]$, and $h_{A}(n)-h_{A}(n-1)=n+1$ for all $n \geq 0$. Conversely, suppose that $M$ has the Hilbert function as mentioned, and let $M$ have a minimal projective resolution given by (2.1). Then $d=h_{M}(0)=\#\left\{i_{\nu} \mid i_{\nu}=0\right\} \leq$ $r$. But, $r \leq \sum_{\nu=1}^{r}\left(j_{\nu}-i_{\nu}\right)=d[7,2.41]$. Thus $r=d$ and then the condition $\sum_{\nu=1}^{d}\left(j_{\nu}-i_{\nu}\right)=d$ implies, using the fact $j_{\nu}>i_{\nu}[3,3.2]$, that $i_{\nu}=0$ and $j_{\nu}=1$ for all $\nu$.

Proposition 2.4. Let $M$ be a normal A-module of GK-dimension 2 and multiplicity $d$, with Hilbert function $h_{M}$. Then $h_{M}$ is a non-decreasing function. Further, if $M$ is critical, then $h_{M}(n)=d n+c$ for $n \geq d$ (where $c$ is a constant depending only on the type of $M$ ).

Proof. Let $s_{M}(n)$ be the "slope" of the Hilbert function, defined as $s_{M}(n)=$ $h_{M}(n)-h_{M}(n-1)$ for all $n \in \mathbb{Z}$. Since the Hilbert function is additive on exact sequences, so is the slope function. Now let $M$ have a minimal projective resolution as in (2.1). Then,

$$
s_{M}(n)=\sum_{\nu=1}^{r} s_{A\left(-i_{\nu}\right)}(n)-\sum_{\nu=1}^{r} s_{A\left(-j_{\nu}\right)}(n) .
$$

Since $h_{A}(n)=(n+1)(n+2) / 2$ for $n \geq 0$, and 0 for $n<0$, it follows that $s_{A(-i)}(n)=n-i+1$ for $n \geq i$, and 0 for $n<i$. Observe that if $i \leq j$, then $s_{A(-i)}(n) \geq s_{A(-j)}(n)$ for all $n$. Minimality of the resolution (2.1) implies that $i_{\nu}<j_{\nu}$ for all $\nu[3,3.2]$. Now assembling the above facts in (2.3) shows that $s_{M}(n) \geq 0$ for all $n$, so $h_{M}(n)$ is a non-decreasing function. Now suppose $M$ is critical. Then $i_{\nu}, j_{\nu} \leq d$ for all $\nu$ [3, Proof of 3.8]. Thus, the formula (2.3), together with the remarks made above about the slope function, shows that for $n \geq d$

$$
s_{M}(n)=\sum_{\nu=1}^{r}\left(n-i_{\nu}+1\right)-\sum_{\nu=1}^{r}\left(n-j_{\nu}+1\right)=\sum_{\nu=1}^{r}\left(j_{\nu}-i_{\nu}\right)=d,
$$

where the last equality is also a consequence of the minimality of the resolution: $[7,2.41],[3,3.2]$. Now the fact $s_{M}(n)=d$ for all $n \geq d$ clearly implies that 
$h_{M}(n)=d n+c$ for $n \geq d$ with an appropriate choice of the constant $c$ (depending on the type of $M$ ).

\section{The Divisor of A MOdule}

From here on we assume that $A$ satisfies the hypotheses of Theorem 1.1.

Recall that $A$ contains a certain canonical central element $g$ in degree three such that $B=A / g A$ is isomorphic to the twisted homogeneous coordinate ring $B(E, \sigma, \mathcal{L})[6],[8]$. Hence there is a category equivalence [8]

$$
(\mathrm{gr}-B) /(\mathrm{gr}-B)_{0} \rightarrow \operatorname{coh}(E): M \mapsto \tilde{M}
$$

reducing the GK-dimension by one. Here $\operatorname{coh}(E)$ denotes the category of coherent $\mathcal{O}_{E}$-modules. The inverse to $(\tilde{-})$ is given by a functor $\Gamma_{*}$ which is defined by

$$
\Gamma_{*}(\mathcal{M})=\oplus_{n \in \mathbb{Z}} \Gamma\left(E, s^{n} \mathcal{M}\right)
$$

where $s \mathcal{M}=\sigma_{*}\left(\mathcal{M} \otimes_{\mathcal{O}_{E}} \mathcal{L}\right)$. Note that, even if $\mathcal{M}$ is coherent, $\Gamma_{*}(\mathcal{M})$ will not always be finitely generated. However all $\Gamma_{*}(\mathcal{M})_{\geq_{n}}$ are finitely generated. In particular $\Gamma_{*}(\mathcal{M})$ is finitely generated when considered modulo $(\mathrm{gr}-B)_{0}$. Using the functor $\Gamma_{*}$ one obtains

$$
M(1)^{\sim}=\sigma_{*}\left(\tilde{M} \otimes_{\mathcal{O}_{E}} \mathcal{L}\right) .
$$

For an $A$-module $M$, the $g$-torsion submodule of $M$ is the graded submodule whose elements are annihilated by a power of $g$. We say that $M$ is $g$-torsion-free (resp. $g$-torsion) if the $g$-torsion submodule of $M$ is 0 (resp. $M$ ).

A cyclic graded $A$-module $N$ generated in degree 0 such that $\operatorname{dim} N_{i}=1$ for all $i$ is called a point module. It is shown in [6], [7] that in the elliptic case all point modules are annihilated by $g$. In fact one has [6], [7]:

Proposition 3.1. The map $p \mapsto \Gamma_{*}(k(p))_{\geq 0}$ defines a bijection between the points of $E$ and the point modules of $A$.

In the sequel we will denote the point module corresponding to $p \in E$ by $N_{p}$. Thus $\tilde{N}_{p}=k(p)$. From (3.3) it follows that

$$
N_{p}(1)_{\geq 1}=N_{\sigma p}
$$

To a $g$-torsion-free $A$-module $M$ of GK-dimension 2, we now associate a divisor $\operatorname{Div}(M)$ on $E$. For such a module $M, M / M g$ is a $B$-module of GK-dimension 1 , so $(M / M g)^{\sim}$ is a finite-dimensional $\mathcal{O}_{E}$-module which corresponds to a divisor on $E$. We denote this divisor by $\operatorname{Div}(M)$. It is easy to check that $\operatorname{Div}(M)$ coincides with another $\operatorname{divisor} \operatorname{div}(M)$ as defined in $[3, \S 3]$ for normal $A$-modules of GK-dimension 2.

If $M$ has multiplicity $d$, then the degree of $\operatorname{Div}(M)$ is $3 d$. In particular $\operatorname{Div}(M) \neq$ 0 iff $M \neq 0$. From (3.3) it follows that

$$
\operatorname{Div}(M(1))=\sigma(\operatorname{Div}(M)) .
$$

The next result is an improvement of [3, 3.35] for $g$-torsion-free modules.

Theorem 3.2. Let $M$ be a g-torsion-free A-module of GK-dimension 2, $M \rightarrow N_{p}$ a non-zero map to a point module $N_{p}$, and $K$ the kernel of this map. Then

$$
\operatorname{Div}(K)=\operatorname{Div}(M)-(p)+\left(\sigma^{-3} p\right) .
$$


Proof. Since the cokernel of the map $M \rightarrow N_{p}$ is finite $k$-dimensional, we have an exact sequence modulo $(\mathrm{gr}-A)_{0}$ :

$$
0 \rightarrow K \rightarrow M \rightarrow N_{p} \rightarrow 0
$$

Tensoring this sequence with $B$, we obtain the following exact sequence modulo $(\operatorname{gr}-A)_{0}$ :

$$
0 \longrightarrow \operatorname{Tor}_{1}^{B}\left(N_{p}, B\right) \longrightarrow K / K g \longrightarrow M / M g \longrightarrow N_{p} \longrightarrow 0
$$

(to see this, observe that $\operatorname{Tor}_{i}^{A}(-, B)$ preserves $\left.(\mathrm{gr}-A)_{0}\right)$.

Using the standard projective resolution for $B$ as an $A$-module we find

$$
\operatorname{Tor}_{1}^{B}\left(N_{p}, B\right)=N_{p}(-3) .
$$

Applying $(\tilde{-})$ to $(3.7)$ and using (3.4) yields

$$
0 \rightarrow k\left(\sigma^{-3} p\right) \rightarrow(K / K g)^{\sim} \rightarrow(M / M g)^{\sim} \rightarrow k(p) \rightarrow 0,
$$

which gives equation (3.6).

Lemma 3.3. Let $M$ be a g-torsion-free A-module of GK-dimension 2.

1. If $M_{<0}=0$ and if $p$ is in the support of $\operatorname{Div}(M)$, then there is a non-zero map $M \rightarrow N_{p}$.

2. If $M$ is normal and $\operatorname{Div}(M)$ has no multiple points, then there is a surjective map from $M$ to a point module.

Proof. 1. Let $\mathcal{M}=(M / M g)^{\sim}$. There is a non-zero map $\mathcal{M} \rightarrow k(p)$, hence a map

$$
M \rightarrow M / M g=(M / M g)_{\geq 0} \rightarrow \Gamma_{*}(\mathcal{M})_{\geq 0} \rightarrow \Gamma_{*}(k(p))_{\geq 0}=N_{p},
$$

which is surjective in high degree.

2. Assume $\operatorname{Div}(M)=p_{1}+\cdots+p_{n}$. Thus $(M / M g)^{\sim}=k\left(p_{1}\right) \oplus \cdots k\left(p_{n}\right)$. Hence

$$
u: M / M g \rightarrow N_{p_{1}} \oplus \cdots \oplus N_{p_{n}}
$$

has finite-dimensional kernel and cokernel. From the Cohen-Macaulayness of $M$ it easily follows that $M / M g$ has no finite-dimensional submodules, so the map $u$ is actually injective. Let $0 \neq m \in M_{0}$. Since $u$ is injective, $u(m)$ has a nonzero component in some $\left(N_{p_{i}}\right)_{0}$. This implies that the composition of $u$ with the projection $N_{p_{1}} \oplus \cdots \oplus N_{p_{n}} \rightarrow N_{p_{i}}$ is a surjective map of $M / M g$ onto $N_{p_{i}}$, hence giving a surjective map $M \rightarrow N_{p_{i}}$.

\section{Proof of Theorem 1.1}

First, it is easy to see that a critical $A$-module is either $g$-torsion or $g$-torsion-free [2, Corollary 2.5]. It is convenient to consider these cases separately.

Proof of Theorem 1.1 ( $g$-torsion-free case). Without loss of generality we may assume that $M$ is Cohen-Macaulay. Shifting if necessary, we may furthermore assume that $M_{<0}=0$.

Let $N$ be some positive number and let $p$ be in the support of $\operatorname{Div}(M)$. Put $K=\operatorname{ker}\left(M \rightarrow N_{p}\right)$. It is easy to see that $K$ is also Cohen-Macaulay and critical. According to (3.6), $p$ in $\operatorname{Div}(M)$ is replaced by $\sigma^{-3} p$ in $\operatorname{Div}(K)$. It is clear that by replacing $M$ by $K$ and repeating this procedure in an intelligent way, we can assure that eventually the following becomes true

$\left(A_{N}\right) \quad \operatorname{Div}(M)$ is reduced and $\sigma^{3 i} p \neq q(0<|i| \leq N)$ for $p, q \in \operatorname{Supp}(\operatorname{Div}(M))$ 
(where we use, of course, that $\sigma$ has infinite order and no fixed points). Now make $M$ normal by shifting. According to (3.5), condition $\left(A_{N}\right)$ is still satisfied.

Inductively, we construct a descending chain of Cohen-Macaulay submodules of $M$

$$
M=M_{0} \supset M_{1} \supset M_{2} \supset \cdots \supset M_{N}
$$

such that $M_{i}$ satisfies $\left(A_{N-i}\right)$. This is done as follows. Suppose we have constructed $M_{i}$. Choose $n$ such that $L=M_{i}(n)$ is normal. By $(3.5) \operatorname{Div}(L)$ is still reduced and hence, by Lemma 3.3, $L$ maps surjectively to a point module $N_{q}$. We put $M_{i+1}=$ $\operatorname{ker}\left(L \rightarrow N_{q}\right)(-n)$. According to (3.5) and (3.6), $M_{i+1}$ will satisfy $\left(A_{N-i-1}\right)$.

The Hilbert function of $M_{i}$ is easy to compute:

$$
h_{M_{i}}(n)=\max \left(h_{M}(n)-i, 0\right) .
$$

According to Proposition 2.4, $h_{M}(n)$ is non-decreasing, and

$$
h_{M}(n)=d n+c_{M} \quad \text { for } n \geq d,
$$

where $c_{M}$ is a constant depending only on the type of $M$. By Theorem 2.2, for a fixed multiplicity $d$, there are only a finite number of possibilities for $c_{M}$. Choose $N=\max _{M}\left(d^{2}+c_{M}\right)$. Applying (4.1) with $i=d^{2}+c_{M}$ we find

$$
h_{M_{i}}(n)=\max (d(n-d), 0) .
$$

Hence by Lemma 2.3, it follows that $M_{i}(d+1)$ has a resolution of the form (1.1).

Proof of Theorem 1.1 ( $g$-torsion case). From the category equivalence (3.1), it follows immediately that all $g$-torsion critical $A$-modules of GK-dimension 2 are GKequivalent. To find a Cohen-Macaulay one which has a resolution as in (1.1), we have to find one which has the correct Hilbert series (according to Lemma 2.3). To do this we use the inverse $\Gamma_{*}$ to $(\tilde{\sim})(3.2)$.

Just as in the commutative case, it is easy to see (using the local cohomology criterion) that if $\mathcal{M}$ is torsion free, then $\Gamma_{*}(\mathcal{M})$ is Cohen-Macaulay. See [4] for foundations.

Let $\mathcal{M}$ be a line bundle of degree 3, not isomorphic to $\sigma_{*} \mathcal{L}$. By Riemann-Roch it is easy to see that $\Gamma_{*}(\mathcal{M})$ has the correct Hilbert series.

\section{REFERENCES}

[1] K. Ajitabh, Modules over regular algebras and quantum planes, Ph.D. thesis, MIT, 1994.

[2] _ Residue complex for three-dimensional Sklyanin algebras, to appear in Adv. in Math.

[3] $\_$, Modules over elliptic algebras and quantum planes, Proc. London Math. Soc. (3) 72 (1996), 567-587. MR 97a:16049

[4] M. Artin and J. J. Zhang, Noncommutative projective schemes, Adv. in Math. 109 (1994), no. 2, 228-287. MR 96a:14004

[5] M. Artin and W. Schelter, Graded algebras of global dimension 3, Adv. in Math. 66 (1987), 171-216. MR 88k:16003

[6] M. Artin, J. Tate, and M. Van den Bergh, Some algebras associated to automorphisms of elliptic curves, The Grothendieck Festschrift, vol. 1, Birkhäuser, 1990, pp. 33-85. MR 92e:14002

[7] _ Modules over regular algebras of dimension 3, Invent. Math. 106 (1991), 335-388. MR 93e: 16055

[8] M. Artin and M. Van den Bergh, Twisted homogeneous coordinate rings, J. Algebra 133 (1990), 249-271. MR 91k:14003 
[9] T. Levasseur, Some properties of non-commutative regular rings, Glasgow Math. J. 34 (1992), 277-300. MR 93k:16045

[10] A. Yekutieli, The residue complex of a non-commutative graded algebra, J. Algebra 186 (1996), 522-543. CMP 97:05

Department of Mathematics, Florida International University, University Park, Miami, FloRida 33199

E-mail address: ajitabhk@zeus.fiu.edu

Departement Wni, Limburgs Universitair Centrum, Universitaire Campus, 3590 Diepenbeek, Belgium

E-mail address: vdbergh@luc.ac.be 